\title{
Electron beam density measurements in shock waves in argon
}

\author{
By B. SCHMIDT $\dagger$ \\ Californiz Institute of Technology
}

(Received 29 August 1968)

\begin{abstract}
An electron beam technique was used to measure density profiles of strong shock waves in argon with high accuracy. The experimental results are compared with the results of theoretical models. Of the models that are available in enough detail for comparison, the best agreement with experiment is shown by the direct simulation Monte-Carlo method (Bird 1968), assuming an inverse 12th power molecular interaction force law. It is shown that the density maximum slope thickness is not sufficient for a detailed description of the shock wave structure.
\end{abstract}

\section{Introduction}

Shock wave structure has attracted a considerable amount of interest because the normal shock wave is the simplest flow in which large departures from equilibrium occur. The flow is one-dimensional, boundary effects are absent, and initial and final states are equilibrium states connected by simple laws.

In order to make a detailed comparison of shock wave structure with the predictions of various theoretical models, more accurate experiments than have been previously reported are required. In the present work sufficiently accurate profiles are presented for shock waves in argon at shock Mach numbers between $2 \cdot 8$ and 8.

Shock wave thickness fails to give sufficiently detailed information about structure; calculated and measured profiles may differ considerably, but the thickness can be the same. It has been found that, judged by thickness, the Mott-Smith method gives good agreement with experiment. However comparison of the profiles shows that the symmetric result of the Mott-Smith model disagrees with the asymmetric result of the experiment for strong shock waves.

Unfortunately, enough information for comparison is available for only a limited number of theoretical models. Of the models that have been compared with the present experiments the best agreement is given by a direct simulation Monte-Carlo method developed by Bird (1968).

\section{Apparatus and experimental procedure}

The experiments have been performed in the 17 in. shock tube at California Institute of Technology. The tube is made of $43.43 \mathrm{~cm}(17 \cdot 1 \mathrm{in}$.) stainless steel

$\dagger$ Present address: University of Karlsruhe, Karlsruhe, Germany. 
tubing and consists of a $3.82 \mathrm{~m}$ (8.78 diameter) long driver section and a $21.3 \mathrm{~m}$ ( 49 diameter) long driven section. The electron beam is located 48.5 diameters downstream of the diaphragm and $20 \mathrm{~cm}$ from the end wall.

Figure 1 shows the arrangement of the electron beam apparatus. The main concepts in the design of the apparatus have been, first, a rigid, self-supporting

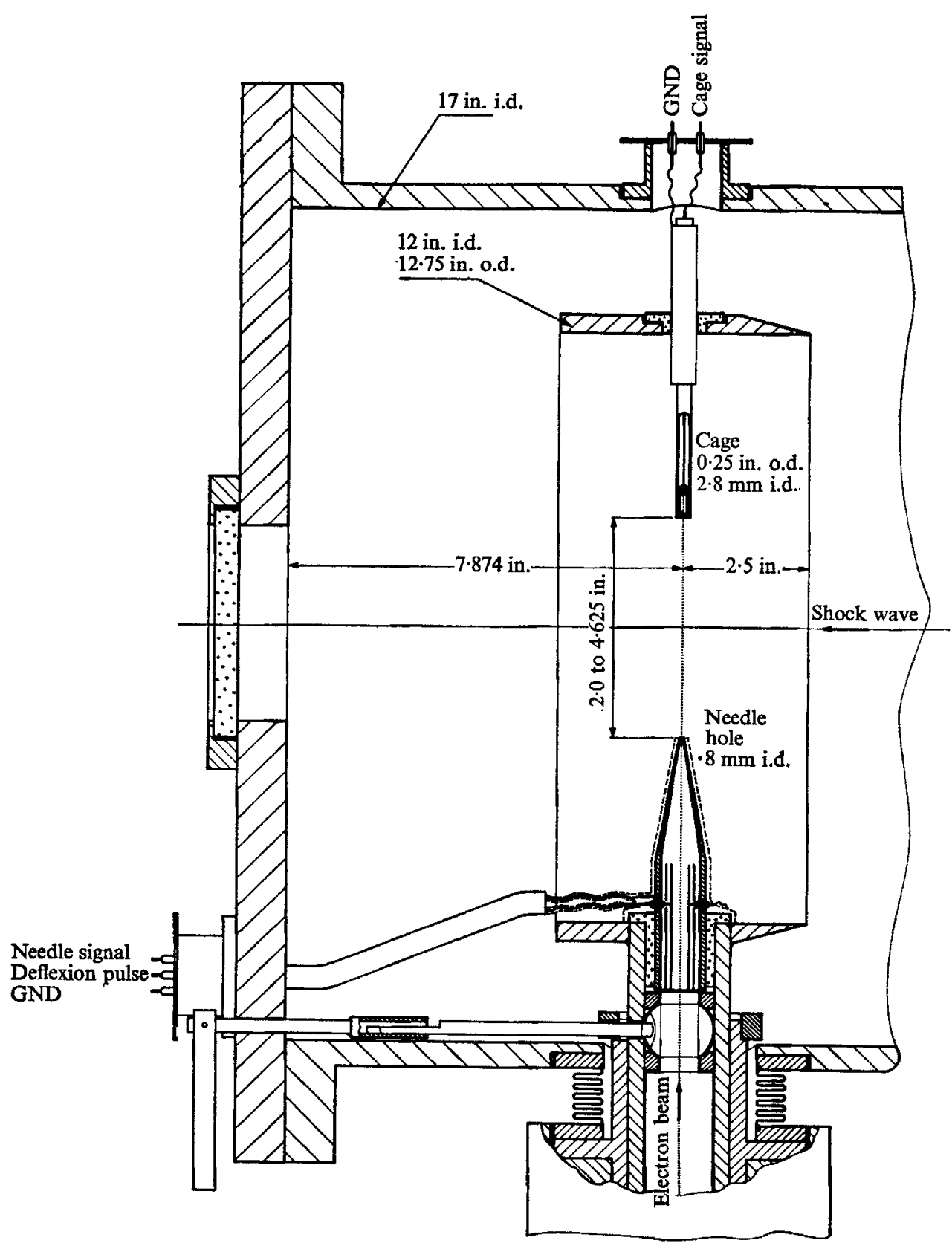

FIGURm 1. Electron beam apparatus. 
structure that is uncoupled from the shock tube and, second, an alignment procedure that is guided by current measurements only, without visual observation of the beam. The design benefits from experience obtained with a similar apparatus designed by Russell (1965, pp. 265-76).

The electron beam is generated by a commercial T.V. picture tube gun $\dagger$ and is injected into the shock tube through a $0.8 \mathrm{~mm}$ hole in the cone tip ('needle' in figure 1). A small Faraday cage with $2.8 \mathrm{~mm}$ i.d., located opposite to the cone, collects the beam.

The cone tip and the Faraday cage are arranged symmetrically about the shock tube axis and protrude into the shock tube far from the walls. Thus, the beam traverses only the least bowed part of the shock wave. The gap between cone tip and cage (sampling length) is variable between 50 and $118 \mathrm{~mm}$.

Cage and cone are supported by a $30.48 \mathrm{~cm}$ (12 in.) i.d. ring, which is arranged concentric to the shock tube. The ring is wedge-shaped on the upstream side. A stiff tube, the stem, connects the $12 \mathrm{in}$. ring with the gun chamber block located outside of the shock tube. The gun chamber can be shut off from the shock tube by a ball valve inside the stem, operated from outside the shock tube. The entire apparatus is supported independently of the shock tube and the only connexion between the two is a very flexible bellows. The low pressure required inside the gun chamber is maintained by an oil diffusion pump. In the worst case $\left(p_{1}=100 \mu\right.$, ball valve open) the pressure is below $0 \cdot 1 \mu$.

The gun generates an electron beam $f$ of roughly $1.0 \mathrm{~mm}$ diameter that is reduced by the injection hole to $0.8 \mathrm{~mm}$ diameter. A beam spreading over the sampling length has not been observed.

Faraday cage and injection cone are insulated electrically and connected to ground through a $1000 \Omega$ resistor. Therefore, the currents to cage and cone generate voltage signals which are fed separately over preamplifier (Philbrick $\mathrm{P} 25 \mathrm{AH}$ ) into an array of oscilloscopes (Tektronix 555 and 535).

Before every shot the shock tube is pumped to $0 \cdot 1 \mu\left(1 \mu=10^{-3} \mathrm{Torr}\right)$, flushed with the test gas at $50 \mu$, and again pumped to $0.1 \mu$. Then the test gas is released into the shock tube from a calibrated volume of one thousandth of the driven section volume. The pressure inside the calibrated volume is controlled by a precision pressure gauge (Wallace \& Tiernan, 0-50Torr abs.) and the test section pressure is checked occasionally with a McLeod vacuum meter.

The shock wave velocity is measured with thin film heat-transfer gauges arranged $50 \mathrm{~cm}$ apart, with the downstream gauge 1.45 diameters upstream of the electron beam. The signals from the gauges are amplified and fed into a time interval meter (Hewlett Packard counter $5233 \mathrm{~L}$ ).

To record the zero current level for the cage signal, and the beam intensity at the point of injection, the electron beam is deflected electrostatically. Approximately $50 \mu$ s after passage of the shock wave a square wave signal is applied to deflexion plates inside the injection cone (cf. figure 3).

A few milliseconds after the shock wave passes the measuring station, the beam

† Superior Electronics Laboratories, N.J., type SE 110 E.

\$ The measurements are made with $15 \mathrm{kV}$ acceleration voltage and $90-120 \mu \mathrm{a}$ beam current. 
high voltage is switched off automatically and the ball valve isolates the gun chamber from the shock tube. These measures increase the lifetime of the gun from only a few runs to over eighty.

\section{Calibration and data reduction}

In order to obtain a calibration under static conditions the beam is modulated with a square wave that is just strong enough to deflect it from the injection hole. The subsequent cage and cone signals are displayed on a synchronized dual beam oscilloscope and recorded photographically. The traces are two synchronous square waves with amplitudes proportional to (i) the beam current $I_{0}$ at the injection point and (ii) the beam current $I$ at the cage after attenuation by scattering over the sampling length. If the gain ratio of the two channels is unity the ratio of the square wave amplitudes is equal to the current ratio $I_{0} / I$.

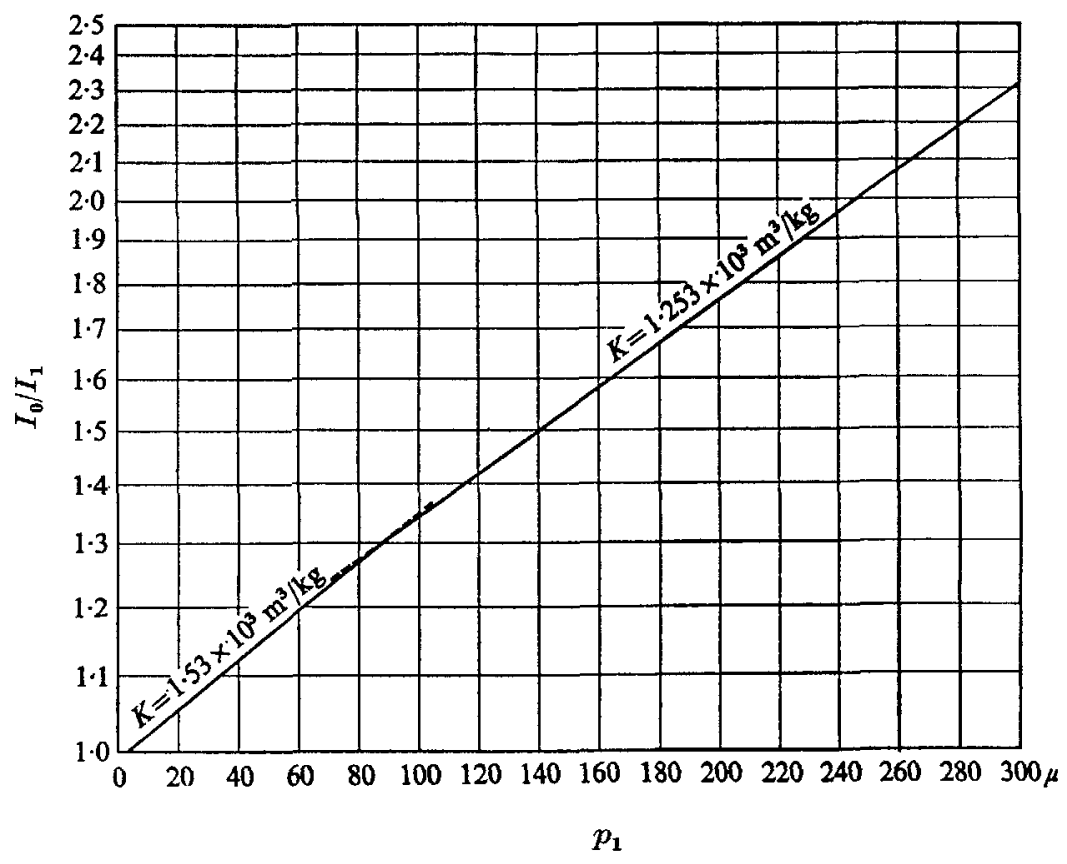

FigUre 2. Electron beam calibration. Argon, 3 in. gap, 15,000 V.

If the exponential scattering law $I / I_{0}=\exp (-K \rho)$ holds, where $\rho=$ density and $K=$ proportionality factor, then $\log I_{0} / I$ plotted versus $\rho$ should be a straight line. The curve shown in figure 2 is the average of several calibrations. Surprisingly, it consists of two straight lines with different slopes, the transition occurring near $p=90 \mu$. A reasonable explanation for this behaviour is not known yet.

It has been found unnecessary to use the static calibration to reduce the shock 
wave data. For, since the values, $\rho_{1}, \rho_{2}, I_{1}$ and $I_{2} \dagger$ corresponding to the equilibrium states far upstream and far downstream of the shock wave are known, the measured current can be converted to density directly using the scattering law. It has been found that, within the scatter and for $p_{1} \leqslant 25 \mu$, the dynamic calibration constant checks the lower range static calibration constant.
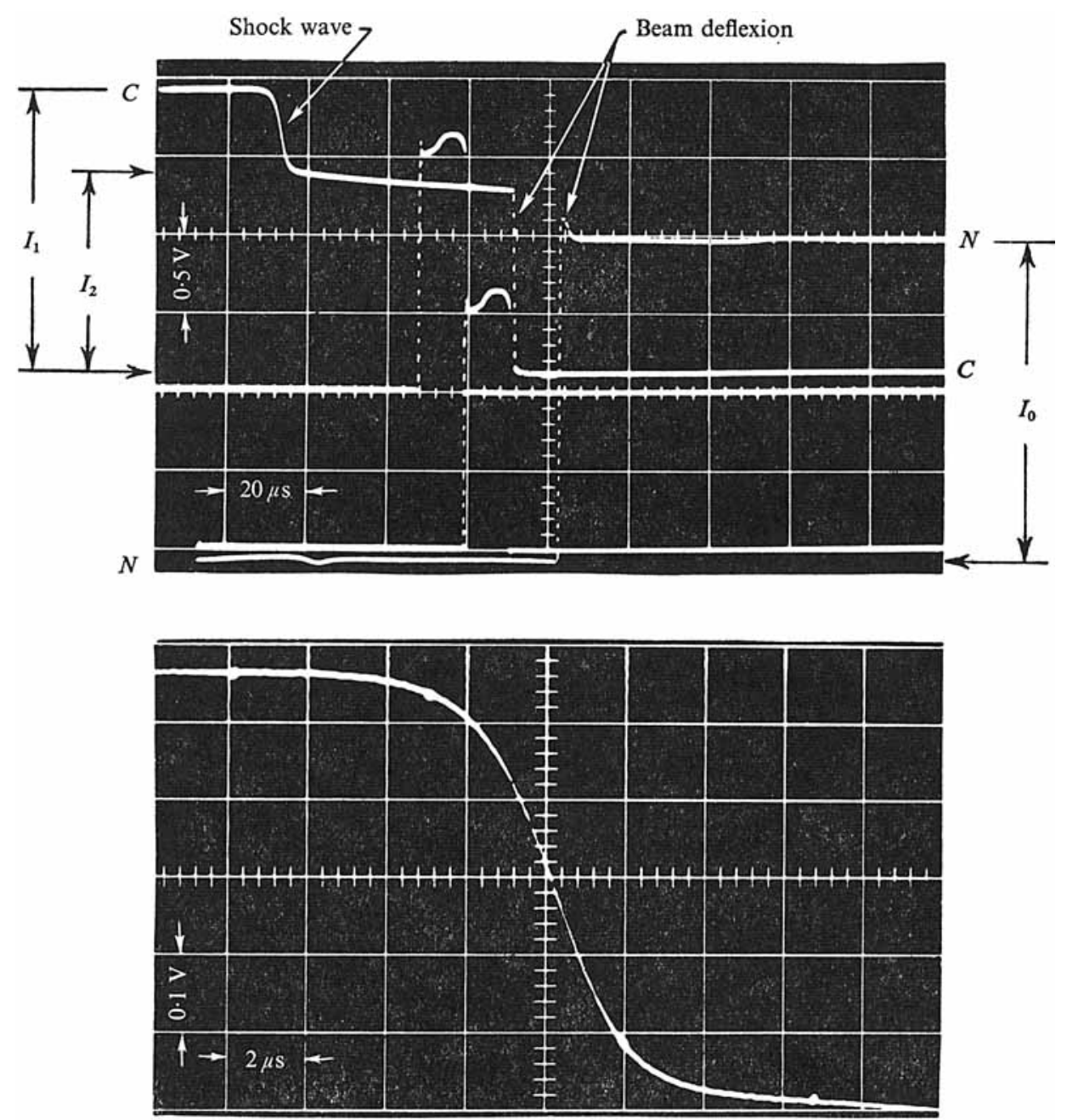

Figure 3. Typical oscilloscope traces. $M_{s}=6 \cdot 07, p_{1}=25 \mu$, argon.

Figure 3 shows typical oscilloscope traces for one run. The cage and the cone signals are marked by $C$ and $N$ ( $N$ is shifted half a division horizontally to avoid signal overlapping). The $10 \mu$ s long distorted square waves on the top picture are used to determine the gain ratio of the cage and the cone circuits. The bottom picture shows the shock wave part of the cage signal enlarged. The bright dots

$\dagger$ The usual shock notation will be used throughout this report. 1 = equilibrium state upstream of the shock wave, 2 = equilibrium state downstream of the shock wave. 
on the trace are time marks from a quartz oscillator controlled time mark generator.

Figure 4 shows how the data are obtained from the bottom picture of figure 3 (the zero current level is obtained from the top picture). Because all pictures show that the beam current decreases nearly linearly for at least two shock thicknesses downstream of the shock wave (cf. error estimation), the end of the wave and therefore the location of point ' 2 ' is indistinct. In this work point 2 has been assigned to the point at which the curved trace merges into the linear decrease (figure 4). This method results in much less scatter than using $I_{0}$ and $I_{1}$, determined on the top picture of figure 3 , to calculate $I_{2}$ and therefore the position of point 2 ; the tolerance $\epsilon$ in figure 4 indicates the error in $I_{2}$ that would result if an error of only $\pm 2.5 \%$ is made reading $I_{0}$ from figure 3 .

Conversion of the time $t$ to the distance $x$ for the purpose of plotting density profiles is accomplished by multiplying $t$ with the measured shock speed $u_{8}$.

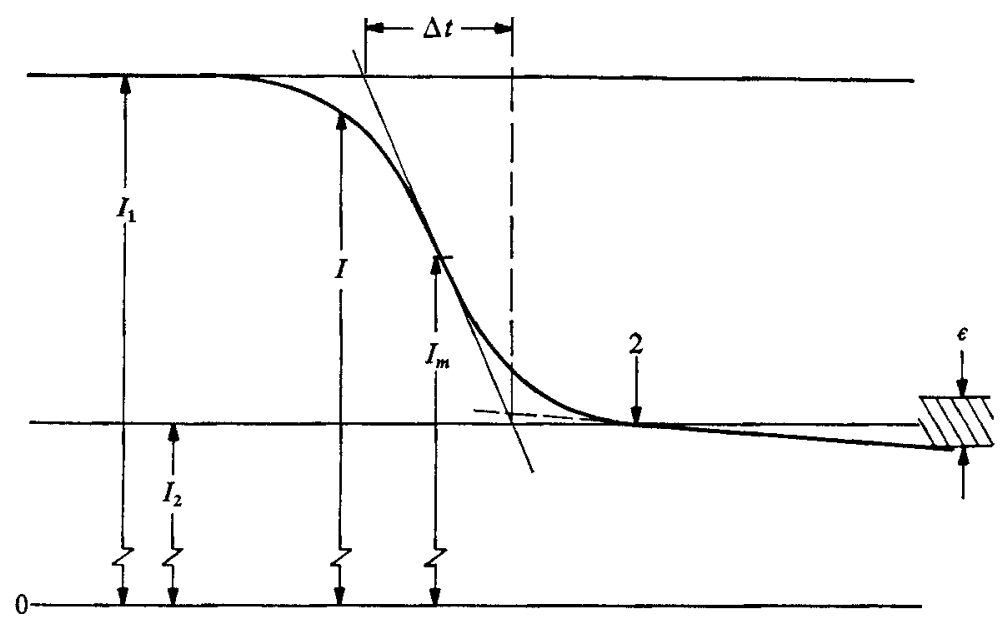

FIGURE 4. $\frac{\rho-\rho_{1}}{\rho_{8}-\rho_{1}}=\frac{\log _{e}\left(I_{1} / I\right)}{\log _{e}\left(I_{1} / I_{2}\right)}, \frac{\lambda_{1}}{\delta_{s}}=\frac{1}{\log _{e}\left(I_{1} / I_{2}\right)} \frac{I_{1}-I_{2}}{I_{m}} \frac{\lambda_{1}}{\Delta t \cdot u_{s}}$.

\section{Error estimation}

An error estimate indicates that, of the quantities derived from the measurements, the largest errors are associated with the mean free path $\lambda_{1}, \dagger$ the shock thickness $\delta_{8} \ddagger$ and the normalized density $R=\left(\rho-\rho_{1}\right) /\left(\rho_{2}-\rho_{1}\right)$. In the worst case, $\lambda_{1}$ can be wrong by $\pm 4 \cdot 6 \%, \delta_{s}$ by $\pm 3 \cdot 3 \%$ and $R$ by $\pm 3 \%$. The main contributions to these errors are connected with the determination of $p_{1}$ and the reading error of $\Delta t$ (cf. figure 4 ) and cage current.

Other factors that introduce errors are the finite rise time $\tau$ of the cage circuit ( $\tau=0 \cdot 1 \mu \mathrm{s}$, measured), the finite ratio of the electron beam diameter $d$ to the

† $\lambda_{1}=\frac{16}{\mathrm{~g}} \sqrt{ }(\gamma / 2 \pi) \mu_{1} / \rho_{1} a_{1}$ where $\mu=$ viscosity, $\rho=$ density and $a=$ speed of sound $\lambda_{1}=5.49 \mathrm{~cm}$ for $\gamma=\frac{5}{3}, T_{1}=300^{\circ} \mathrm{K}$ and $p_{1}=1 \mu$.

$\ddagger \delta_{\mathrm{s}}=u_{\mathrm{s}} \Delta t$ where $u_{\mathrm{t}}=$ shock wave speed and $\Delta t$ is defined in figure 4 . 
shock thickness $\delta_{s}$, the measurement of shock speed, and shock curvature. All these errors amount to less than $1 \%$.

As already mentioned, a continuous but small density rise downstream of the shock wave is observed in all measured profiles. This is partly caused by the boundary layer on the shock tube wall behind the shock wave, whose displacement velocity causes the density to increase downstream of the shock wave and the shock wave to bulge forward.

Calculating the density rise behind the shock wave using the measured curvature (Liepmann \& Bowman 1964) gives a smaller density rise than measured, but with the same dependency; the density rise is nearly independent of shock strength and becomes smaller with increasing $p_{1}$.

Furthermore, it is observed that the deviations behind the shock wave depend (inversely) on the sampling length. These distortions may have been caused by aerodynamical disturbances generated by the cone and the cage, or by a changing inflow pattern at the injection hole. They show the same tendency with varying $p_{1}$ and $M_{8}$ as those caused by the boundary layer. A reasonable explanation for effects which depend on sampling length is not known yet.

\section{Results}

The results of the measurements are shown in figure 5. The profiles are the averages of the results of at least nine runs at various $p_{1}$ and fixed $M_{s}$. The extent of scatter at one Mach number is indicated on figure 6. The profile for $M_{s}=2 \cdot 8$ is not shown on figure 5; except for a shorter foot on the low density side it coincides with the profile for $M_{s}=6$.

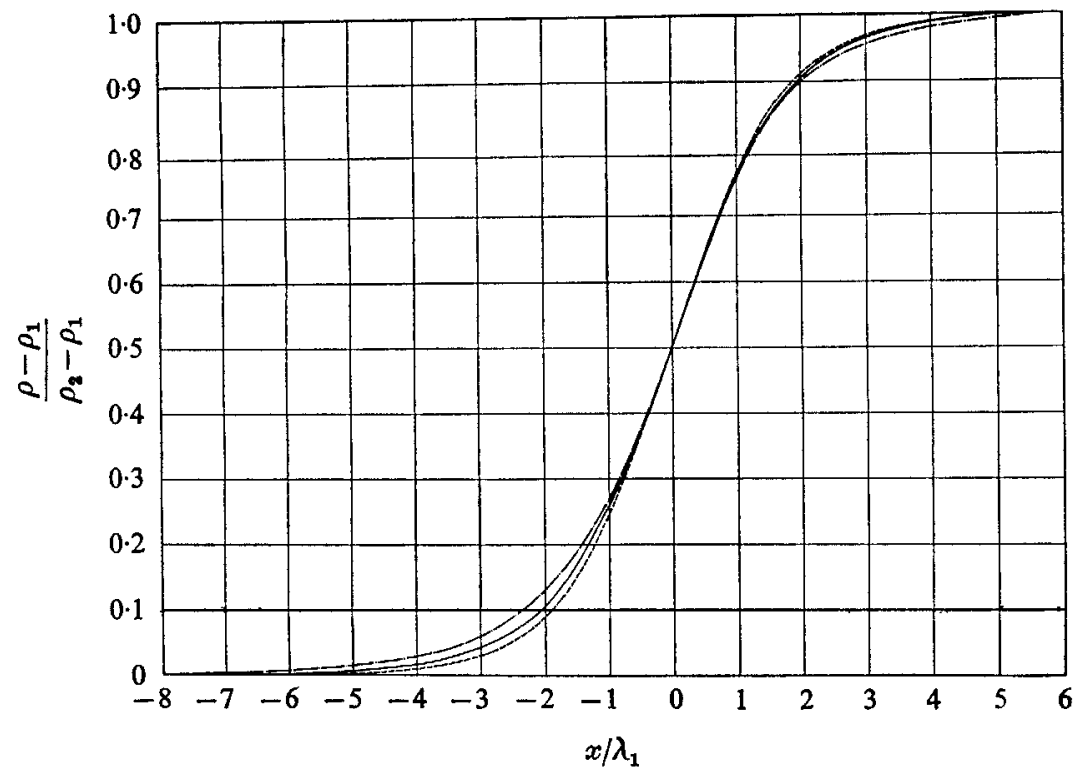

Figure 5. ---, $M_{\mathrm{s}}=4 ;-, M_{\mathrm{s}}=6 ;-\cdot-\cdot, M_{\mathrm{a}}=8$. 
The effect of $M_{s}$ on the profiles is small, the only significant differences being on the low density side of the wave (the deviations observed in the high density part of the profiles are within experimental accuracy). Thus, increasing shock strength causes the profiles to become more asymmetric.

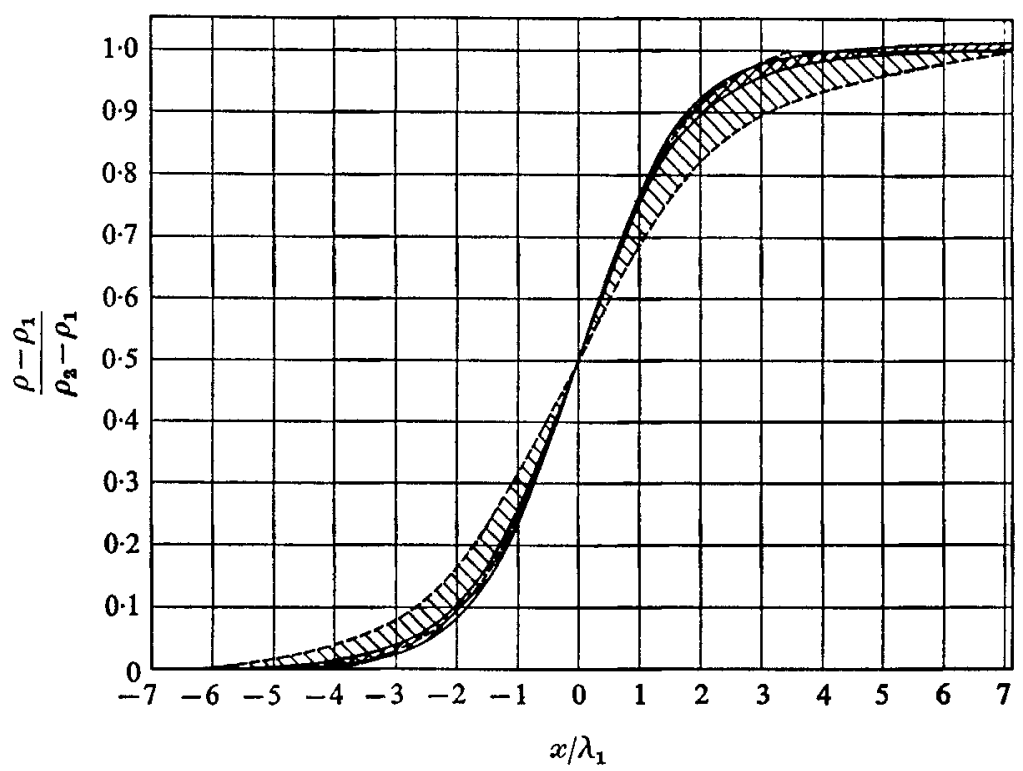

FiguRE 6. $M_{s}=4$, argon, Q7, present experiments; II, Schultz-Grunow \& Frohn (1965).

Experimental results for comparison of full profiles are available only in the paper of Schultz-Grunow \& Frohn (1965, pp. 250-64). As figure 6 shows, the scatter of their experiments is much larger than ours, and therefore important details are lost.

A comparison of some theoretical results with the experiments is shown in figures $8(a),(b)$ and $(c)$. The selection of theories is not complete because detailed information is available for only a limited number of theoretical models. Very good agreement is obtained with the direct simulation Monte-Carlo method of Bird (1968), using an inverse 12th power intermolecular force law $\left(F \sim r^{-\nu}\right.$, $\nu=12, r=$ distance of colliding molecules). The difference in agreement between $\nu=12$ and $\nu=11$ was easily distinguishable. Bird gives an estimated accuracy for his results of $1 \%$.

$\nu=12$ corresponds to a viscosity-temperature relation $\mu \sim T^{0.68}$. This is the same dependence that has been measured in recent heat transfer experiments (Matula 1967) covering the same temperature range $\left(300-6000^{\circ} \mathrm{K}\right)$. In this work the same viscosity-temperature law is used in all the theoretical models.

The agreement with the other models is not as good, and, aside from discrepancies in predicted shock thickness, the most important area of disagreement 
is the extent of asymmetry evidenced by the profiles. A sensitive measure of asymmetry used here for quantitative comparison is

$$
Q=\frac{\int_{-\infty}^{0} R(z) d z}{\int_{0}^{+\infty}(1-R(z)) d z}
$$

where $z=x / \lambda_{1}, R(z)=\left(\rho(z)-\rho_{1}\right) /\left(\rho_{2}-\rho_{1}\right)$ and $R(0)=0 \cdot 5$. The results for different theoretical models and the experiments are shown in figure 7. The NavierStokes equations and the BGK model (Liepmann et al. 1962) give too asymmetric a profile. The Mott-Smith method (Muckenfuss 1962) gives a symmetric profile $(Q=1)$ so its disagreement with experiment increases with shock strength.

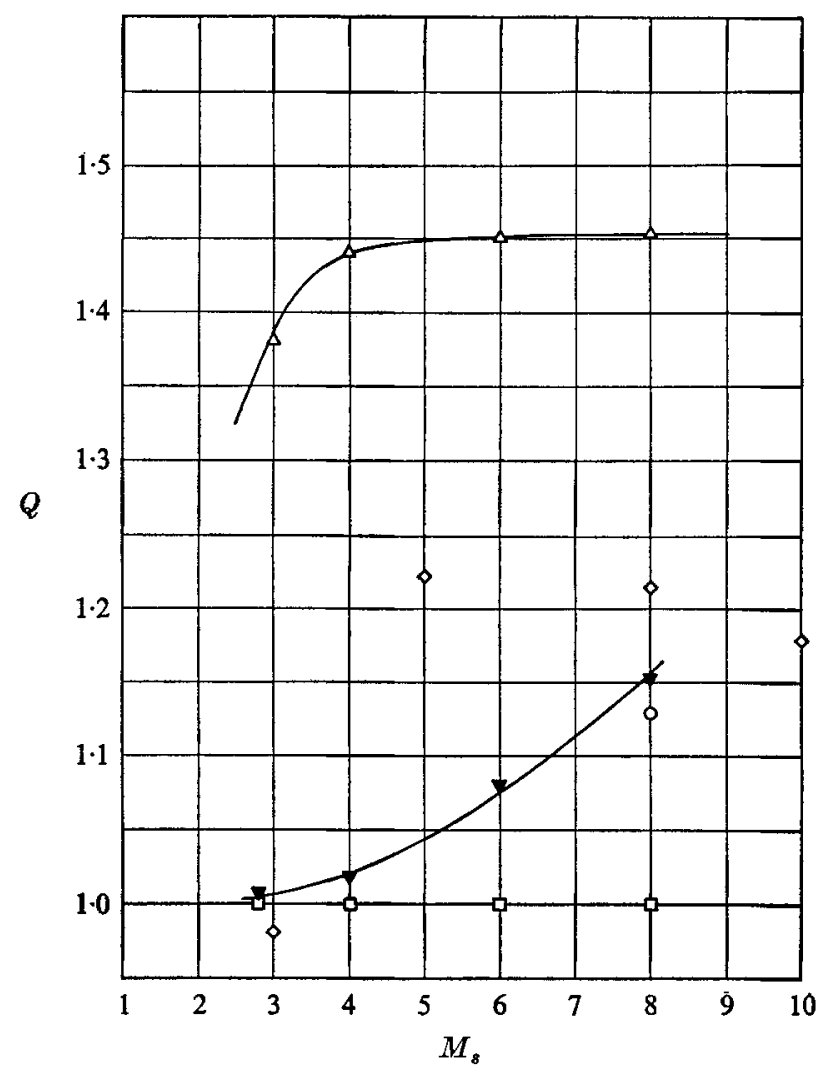

FiguRe 7. $\triangle$, Navier-Stokes; $\diamond, \mathbf{B G K ;} \square$, Mott-Smith; $\mathrm{O}$, Monte-Carlo; $\boldsymbol{\nabla}$, present results.

Many more experimental and theoretical results are available for shock thickness. Although the present experiments do not disagree with previous measurements of shock thickness, the data are shown for completeness in figure 9.

The present data agree in the large with the results of Russell (1965), Camac 


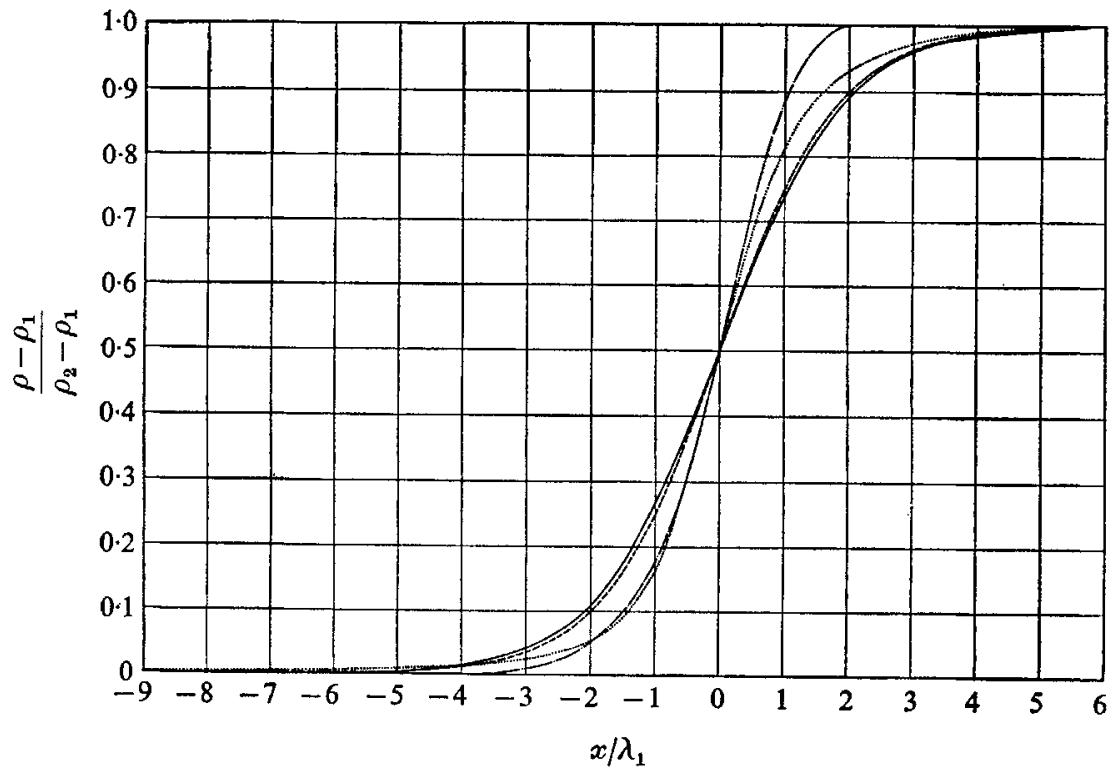

$(a)$

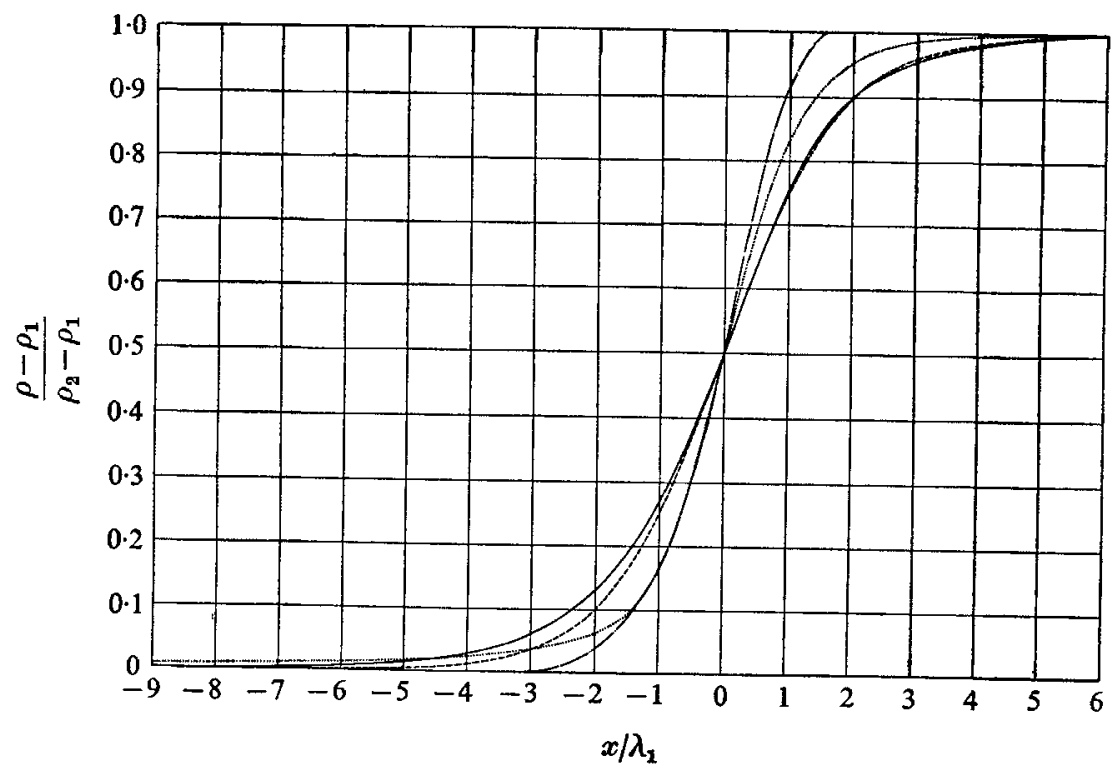

(b)

Figure 8. (a) $M_{s}=2.8, \mu \sim T^{0.68}$. -....., Navier-Stokes; ....., BGK model; ......,

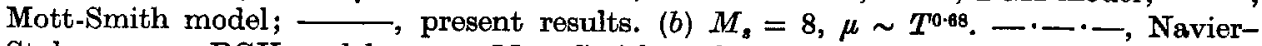
Stokes; ......, BGK model ; -....., Mott-Smith model; — - , present results. 


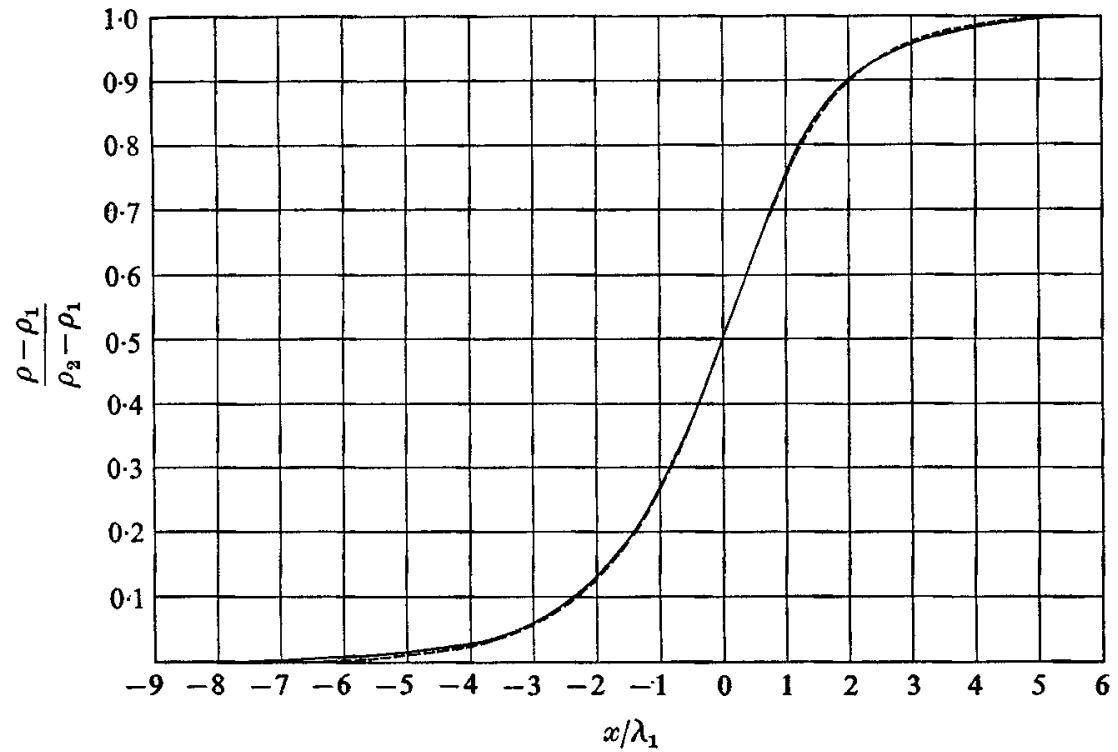

Figure 8 (c) $M_{s}=8 . \longrightarrow$, experiment; ----, Monte-Carlo method, $\nu=12$.

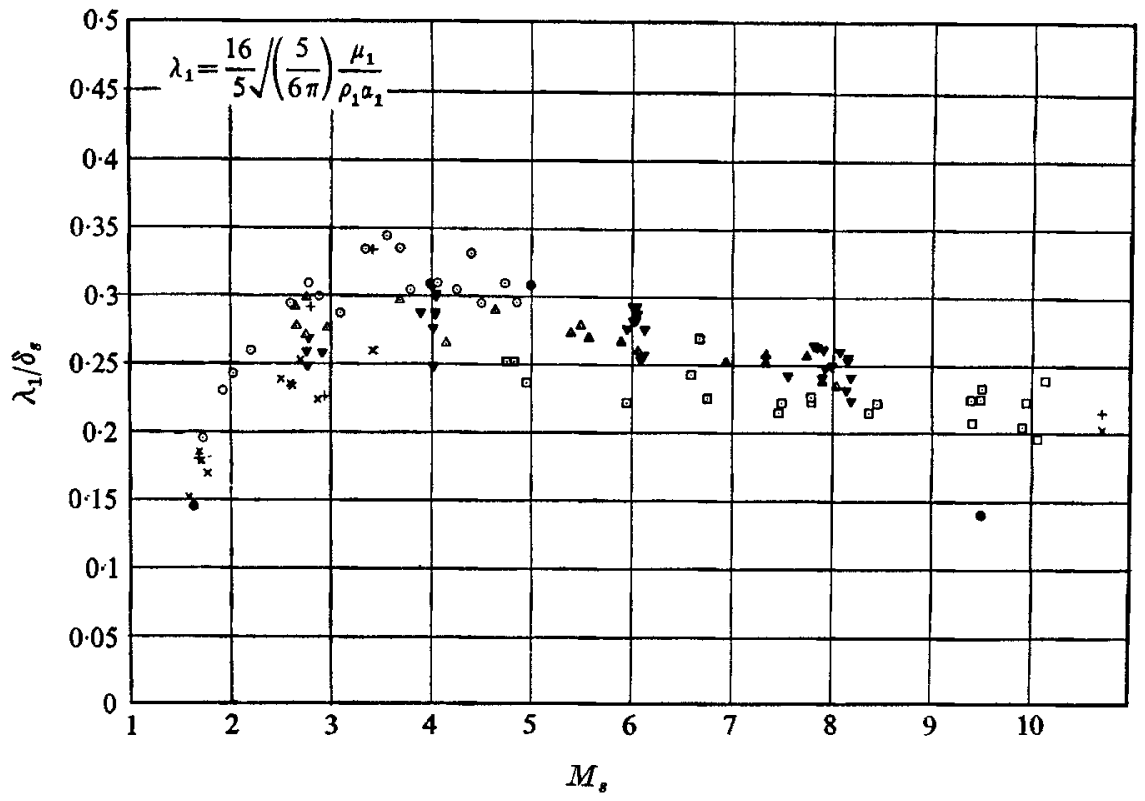

FIGURE 9. $\lambda_{1}=\frac{18}{6} \sqrt{ }(5 / 6 \pi) \mu_{1} / \rho_{1} a_{1} . \boxminus$, Camac; $\odot$, Linzer \& Hornig; $x$, argon, Robben \& Talbot; + , helium, Robben \& Talbot; $\triangle$, Russell; $\bullet$, Schultz-Grunow \& Frohn; present results. 


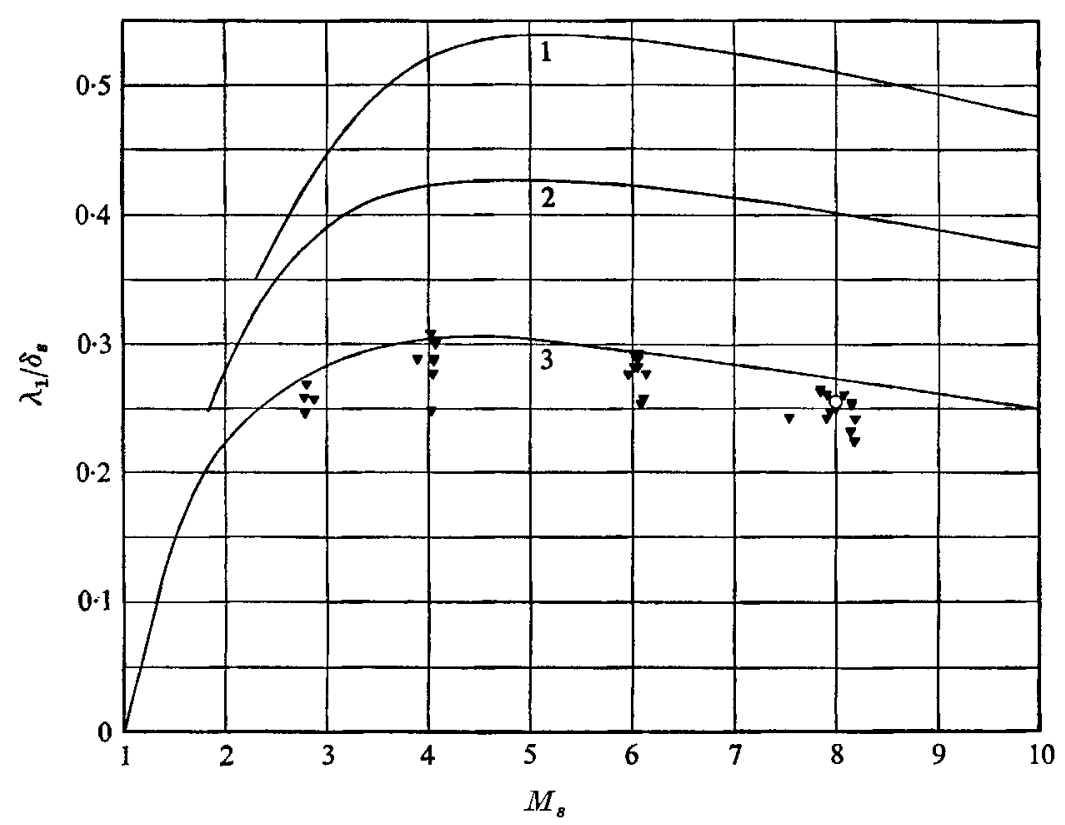

Figure 10. $\mu \sim T^{10.68}$. 1, Navier-Stokes; 2, BGK model; 3, Mott-Smith model; O, Monte-Carlo method; $\mathbf{\gamma}$, present results.

(1965, pp. 240-49) found thicker shock waves ( $\sim 15 \%$ ) for $M_{s}<8$; his data merge with the present ones at $M_{s} \approx 8$. Slightly thinner waves $(\sim 6 \%)$ are reported by Schultz-Grunow \& Frohn (1965) for $M_{s}=4$ and 5. Linzer \& Hornig (1963) found even thinner shock waves $(\sim 12 \%)$. The results of Robben \& Talbot (1966) agree with the present data at $M_{s}=2 \cdot 8$, give thicker shock waves for argon $(\sim 8 \%)$ and thinner ones for helium $(\sim 12 \%)$ at $M_{s}=3.4$ and merge with Camac's results at $M_{s}=10 \cdot 7$.

The measured shock thickness is compared with the model solutions in figure 10. Disagreement with the Navier-Stokes solution and the BGK model are visible here, too. The Mott-Smith method agrees well with the experiment; the differences in the profile do not show up here. This example demonstrates that shock wave thickness fails to reflect important details of shock wave structure.

\section{Conclusion}

It has been shown that a high degree of accuracy is required if the measurement of density profiles is to exhibit important details of shock structure. Of the theoretical models that are available in enough detail for comparison, the best agreement with experiment is given by the direct simulation Monte-Carlo method (Bird 1968), assuming an inverse 12th power molecular interaction force law $\left(\mu \sim T^{0.68}\right)$. Further, it has been shown that thickness is not sufficient for describing details of shock wave structure.

For the present experiments the limits in accuracy have been set by the pressure measurement $\left(p_{1}\right)$ and reading errors of the oscilloscopes. 
The author is greatly indebted to Prof. D. Coles for his helpful suggestions on the design of the electron beam and to Prof. B. Sturtevant for his valuable criticism of the manuscript. Thanks are also due to Prof. H.W. Liepmann for his encouragement and continued interest in the work.

The research employed the Graduate Aeronautical Laboratories, California Institute of Technology $17 \mathrm{in}$. shock tube and auxiliary equipment, which were construeted under NASA Grant NsG 40-60. This work was carried out while the author was in receipt of a NASA International University Fellowship in the Space Sciences.

\section{REFERENCES}

BrrD, G. A. 1968 Proceedings of the Sixth International Symposium on Rarefied Gas Dynamics (in print).

Camac, M. 1965 Rarefied Gas Dynamics, I (Ed. de Leeuw). New York: Academic.

LiepMann, H. W. \& Bowman, R. M. 1964 Phys. Fluids, 7, 2013-15.

Liepmann, H. W., Narasimha, R. \& Chahine, M. 1962 Phys. Fluids, 5, 1313-24.

Linzer, M. \& Hornig, D. F. 1963 Phys. Fluids, 6, 1661-68.

Matula, R. A. 1967 ASME Paper 67.WA/HT-3.

Muckenfuss, C. 1962 Phys. Fluids, 5, 1325-36.

Robben, F. \& Talbot, L. 1966 Phys. Fluids, 9, 633.

Russell, D. 1965 Rarefied Gas Dynamics, I (Ed. de Leeuw). New York: Academic.

Schultz-Grunow, F. \& Frohn, A. 1965 Rarefied Gas Dynamics, I (Ed. de Leeuw). New York: Academic. 